\title{
Beruflich bedingtes allergisches Handekzem durch Euro-Münzen bei vorbestehender Hyperhidrosis manuum
}

\author{
M. Wahlen \\ N. Buhles
}

\author{
Occupational Contact Dermatitis Due to Euro Coins in Patients Suffering from \\ Concurrent Hyperhidrosis of the Palms
}

\section{Zusammenfassung}

Nickel ist das meist verbreitete Allergen in Europa und weltweit, so auch in Euro-Münzen. Münzen werden allerdings nicht zu den Gebrauchsgegenständen, die direkten und langen Kontakt zur Haut haben, gerechnet. Die 1-Euro-Münzen und die 2-EuroMünzen weisen eine Bimetallstruktur auf. In Kombination mit Schweiß entsteht aus diesem Grunde ein sog. „Galvanisches Element“. Durch einen messbaren Stromfluss werden vermehrt Nickelionen freigesetzt. Wir berichten über den Fall eines 55-jährigen Bankkaufmannes in einer Sparkasse, der arbeitskongruent im September 2001 ein dyshidrotisches Handekzem entwickelte. Der Patient wies zudem eine Hyperhidrosis manuum auf. Zuvor hatte der Patient nie an Ekzemen gelitten. Ab Herbst 2001 kam der Patient im Rahmen der Einführung erstmals mit Euro-Münzen in Kontakt. Ein Epikutantest zeigte eine Typ-IV-Sensibilisierung gegenüber Nickel und den Inhaltsstoffen von 1-Euro- und 2-Euro-Münzen.

\section{Abstract}

Nickel is the most prevalent allergen in Europe and world-wide; last but not least in euro-coins. Coins are not regarded as article for daily use with direct and prolonged contact with the skin. The 1- and 2-euro coins show a bimetallic structure. Therefore the coins generate a galvanic element especially in combination with sweat. By this way a high release of nickel due to electricity has been measured. We report on a 55-year old cashier working in a bank. He developed a dyshidrotic hand eczema in September 2001. This patient suffered concurrently of a hyperhidrosis of the palms. In autumn 2001 the patient for the first time had contact to euro-coins. Positive patch test results were obtained against nickel and the contents of 1-euro- and 2-euro coins.

\section{Einleitung}

Das die Typ-IV-Sensibilisierung gegen Nickel-II-Sulfat auslösende Nickelion ist ein ubiquitäres Allergen und das häufigste Kontaktallergen in Europa bzw. weltweit [1]. 10 bis 15\% der Frauen und 2 bis 4\% der Männer leiden an einer Nickelkontaktallergie.

Nickel ist ein häufig verwendetes industrielles Produkt, das als Legierungsbestandteil in vielen Metallen und Edelmetallen zu finden ist. So ergeben sich im beruflichen und privaten Leben zahlreiche Kontaktmöglichkeiten, wobei heutzutage insbeson- dere die Freisetzung von Nickelionen aus vernickelten Gegenständen wie Modeschmuck, Brillengestelle, Knöpfe usw., bei denen intensiver und direkter Hautkontakt gegeben ist, für die hohe Prävalenz von Sensibilisierungen in der Bevölkerung verantwortlich gemacht wird. Die Sensibilisierung erfolgt insbesondere nach Durchstechen der Ohrläppchen [2].

Wichtige Einflussfaktoren für die Auslösung einer Nickelallergie sind das Vorhandensein nickelfreisetzender Materialien, eine ausreichende Kontaktzeit, ein entsprechender Hautkontakt und der Zustand der epidermalen Barriere. Bei bekannter atopischer 
Hautdiathese und stark verminderter Alkaliresistenz ist von einer geschwächten epidermalen Barriere auszugehen [3].

Insbesondere Personen, die ganztägig beruflich mit Münzen in Kontakt kommen, laufen Gefahr allergische Kontaktekzeme auf Nickel zu entwickeln. Gerade im Hinblick auf das Wissen um die Bimetallstruktur und den Nickelanteil der Euro-Münzen scheint ein Handekzem bei Patienten mit bekannter atopischer Hautdiathese, stark verminderter Alkaliresistenz des Hautorgans und einer geschwächten epidermalen Barriere bei bestimmten Berufsgruppen vorprogrammiert zu sein. Diesem Umstand ist im Rahmen der Euro-Einführung der Europäischen Union 2002 nicht Rechnung getragen worden.

\section{Kasuistik}

\section{Anamnese}

Der 55-jährige männliche Patient leitet als Bankkaufmann eine kleine Geschäftsstelle einer Sparkasse mit nur 2 Mitarbeitern. Wegen der spärlichen Besetzung besteht die Notwendigkeit, dass beide Mitarbeiter alle Tätigkeiten durchführen. Dazu gehören Kundenbedienung, Kassierer- und Bildschirmtätigkeiten. Dabei kommt es zu Hautkontakt mit Euro-Münzgeld, Euro-Papiergeld, Thermopapier und allen im Büro vorhanden Papiersorten. Die Verwahrung und Nutzung von Schlüsseln für Tresor und Wertgelasse sowie der Umgang mit Büroklammern ist unerlässlich.

Seit dem Beginn der Ausbildung 1964 bis zum September 2001 war der Patient Haut erscheinungsfrei. Seit September 2001 besteht ein chronisch rezidivierendes Handekzem mit ausgeprägter Verschlechterung bei der beruflichen Tätigkeit als Bankkaufmann/Kassierer. Die Hautveränderungen blieben immer auf den Bereich der Hände begrenzt. Anamnestisch zeigten sich die Ekzeme erstmals im September 2001 bei Anlieferung des EuroMünzgeldes und erstmaligen Hautkontakt zu Euro-Münzen in Form dyshidrosiformer Bläschen im Bereich der Fingerkuppen von Digitum I und II beidseits. Im Verlauf kam es zum zusätzlichen Befall der Fingerkuppen III beidseits. Trotz Anwendung einer Hautschutzsalbe bis zu 10-mal täglich und wiederholten Gebrauchs von externen Steroiden kam es zu keiner Befundbesserung.

Im November 2001 begann der regelmäßige Hautkontakt zu den Münzen im Rahmen des Abpackens von den bekannten 10-EuroMünzen-Starterkits. Mit Beginn des kontinuierlichen Hautkontakts ab Ende Dezember 2001 zeigte sich eine starke Exazerbation der Hautveränderungen mit ausgedehnter Rhagadenbildung, teilweise blutend, im Bereich der Finger I bis III beidseits mit Ausdehnung der Hautveränderungen auf alle Finger. Es traten wiederholt Arbeitsunfähigkeitszeiten auf.

Seit Februar 2002 ist bei dem Patienten eine Typ-IV-Sensibilisierung gegenüber Nickel-II-Sulfat bekannt, die ambulant hautfachärztlich durch Epikutantestungen ermittelt werden konnte. Das Tragen der Brille bei Kurzsichtigkeit und das Tragen der eigenen Uhr stellt sich allerdings als unproblematisch dar. Der Patient wies zudem eine Hyperhidrosis manuum auf.

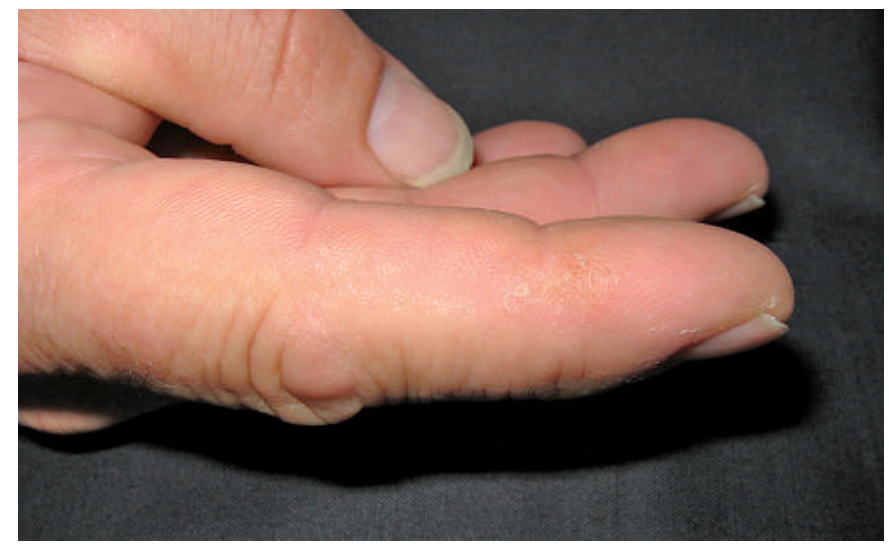

Abb. 1 Es zeigen sich insbesondere am Digitum II der rechten Hand stecknadelkopfgroße Bläschen und ein mittelgradig ausgeprägtes Erythem mit Schuppung.

\section{Hautbefund}

Es zeigen sich bei der Erstvorstellung des Patienten 2003 insbesondere am Digitum II der rechten Hand stecknadelkopfgroße Bläschen und ein mittelgradig ausgeprägtes Erythem mit Schuppung (Abb.1).

\section{Befunde diagnostischer Untersuchungen Epikutantests}

Ein Epikutantest (Fa. Hermal) 2003 am Rücken mittels Epikutantestpflaster nach 24 h, 48 h und 72 h zeigte eine Typ-IV-Sensibilisierung gegenüber Nickel-II-sulfat (5\%). Ein Epikutantest 2003 am Rücken mit 1-Euro- und 2-Euro-Münzen nach $24 \mathrm{~h}, 48 \mathrm{~h}$ und $72 \mathrm{~h}$ zeigte eine Kontaktsensibilisierung gegenüber den Inhaltsstoffen der Münzen. Ein Epikutantest 2005 am Rücken mit 1-Euro-, 2-Euro-, 1-Mark- und 2-Mark-Münzen nach $24 \mathrm{~h}, 48 \mathrm{~h}$ und $72 \mathrm{~h}$ (Abb. 2-5) zeigte ebenfalls eine Kontaktsensibilisierung gegenüber den Inhaltsstoffen aller Münzen.

Gegenüber den mitgebrachten Papierarten (Druckerpapier, FaxThermopapier und Kopierpapier, jeweils mit und ohne Schwärze) konnte 2003 keine Typ-IV-Sensibilisierung nach $24 \mathrm{~h}, 48 \mathrm{~h}$ und $72 \mathrm{~h}$ nachgewiesen werden.

\section{Gesamt-IgE (Fa. Pharmacia) \\ $88 \mathrm{U} / \mathrm{ml}$.}

\section{Erlanger Atopiescore (nach Diepgen)}

18,5 Punkte, somit ist eine 97\%ige Wahrscheinlichkeit einer atopischen Hautdiathese gegeben [4].

\section{Dimethylglyoxim-Test [5]}

Nickelnachweistest (Fa. Squarix biotechnology GmbH, Immuno Select Nickel ) von Uhr, Brille und Sonnenbrille, sowie der 1-Euround 2-Euro- und 1-Mark- und 2-Mark-Münzen:

Kein Nachweis von Nickel an den getesteten Gegenständen.

\section{Hautfunktionstests}

Sebu- und Corneometrie (Fa. Courage u. Khazaka Köln-Germany, Sebumeter SM 810, Corneometer CM 820) im Gesicht, an Handrücken und Unterschenkeln: Zentrale und periphere Sebostase. Leicht verminderte Hydratation des Stratum corneum. 
Abb. 2 Ergebnis der Epikutantestung mit einer 1-EuroMünze nach 72 Stunden am Rücken.

Abb. 3 Ergebnis der Epikutantestung mit einer 2-EuroMünze nach 72 Stunden am Rücken.
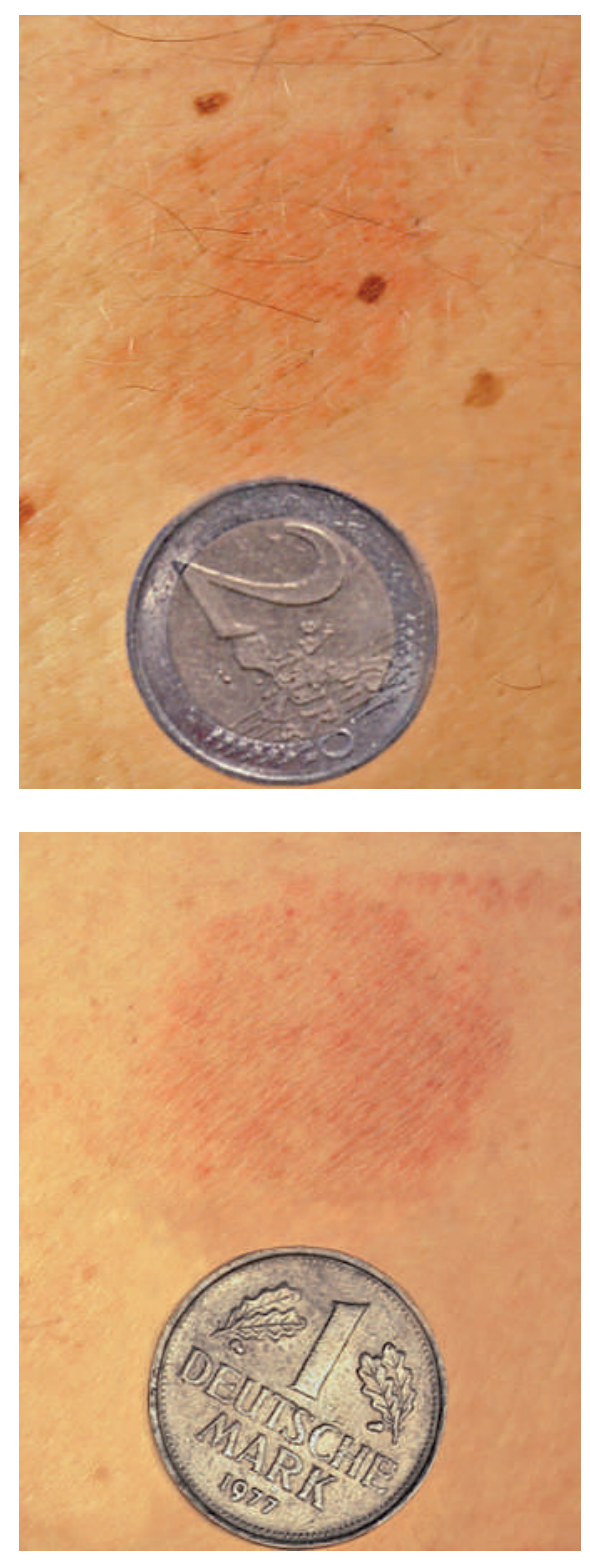

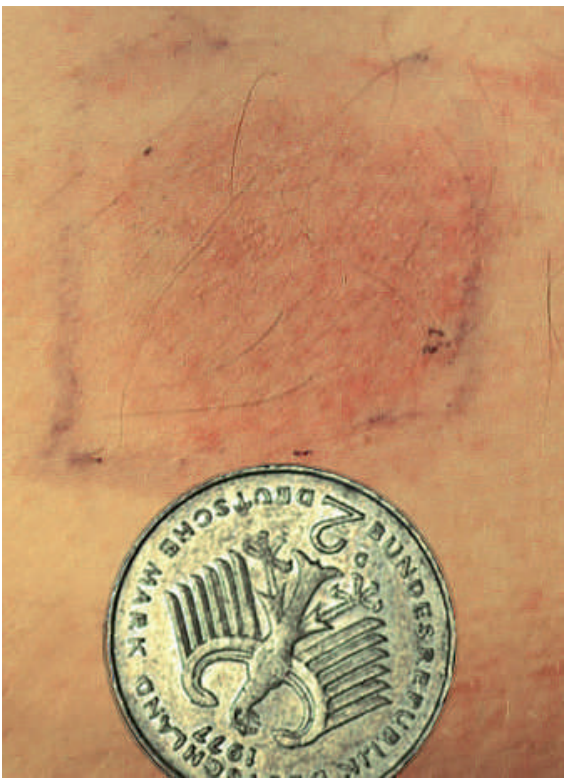

Abb. 5 Ergebnis der Epikutantestung mit einer 2-MarkMünze nach 72 Stunden am Rücken.
Alkalineutralisationstest (nach Burckhardt mit 1/80 normaler $\mathrm{Na-}$ OH-Lösung und 0,5\%iger alkoholischer Phenolphthaleinlösung): Physiologische Alkalineutralisationsfähigkeit des Hautorganes.

Alkaliresistenztest (nach Burckhardt mit 0,5 normaler NaOH-Lösung):

Alkaliresistenz des Hautorgans im getesteten Areal am Unterarm stark vermindert.

Minor'scher Schwitzversuch (Weizenstärke/ Kaliumjodidlsg.): Hinweis auf das Vorliegen einer Hyperhidrosis manuum.

\section{Mykologische Untersuchung}

Kein Nachweis pilztypischer Elemente nativ und kulturell.

\section{Therapie und Verlauf}

Wir sahen den Patienten im Verlauf nach einigen Monaten wieder. Bis dato erfolgte eine weitgehende Vermeidung des Kontaktes zu Hartgeld durch innerbetriebliche Absprachen und Umstrukturierung des Arbeitsablaufes. Allerdings zeigte sich im Rahmen von Urlaubsvertretungen durch vermehrten Münzkontakt eine erneute Exazerbation des Hautzustandes. Unter der Prämisse weiterhin Hartgeldkontakt weitestgehend zu vermeiden, wurde dem Patienten ein Leitungswasseriontophoresegerät für den häuslichen Gebrauch verordnet, um durch weniger Hyperhidrosis an den Händen weniger „Galvanisches Element“ und damit einen besseren Befund an den Händen zu erreichen.

Die Befragung des Patienten nach einem Jahr unter Anwendung der häuslichen Iontophorese ergab, dass die Ekzemschübe deutlich nachgelassen hatten.

Die Anschaffung eines „Geldwechsel-/Zählautomaten“ (analog der Geräte in „Linienbussen“ o. ä.) für die betroffene Bankfiliale zwecks Schaffung eines „leidensgerechten Arbeitsplatzes“ wurde von der Hauptverwaltung der Bank als „nicht banktypisch“ abgelehnt. 
Diskussion

Das Krankheitsbild „cashiers' disease“, bei dem Kassierer aufgrund von nickelhaltigen Münzen Kontaktekzeme entwickeln, ist weitläufig bekannt und häufig publiziert.

Was, wenn nun der Kassierer oder der Bankkaufmann zusätzlich an einer Hyperhidrosis manuum leidet? Bei genauerer Betrachtung des Euro-Münzgeldes ist im Rahmen einer Nickel-II-SulfatSensibilisierung besonders an die bimetallischen 1-Euro-Münzen und 2-Euro-Münzen zu denken.

Die 10-, 20- und 50-Cent-Münzen bestehen aus „Nordischem Gold“, was eine Kupferlegierung darstellt, die zu 89\% aus Kupfer besteht. Die restlichen Metalle sind Aluminium, Zink und Zinn [6]. Die 1-, 2- und 5-Cent-Münzen bestehen aus Stahl mit Kupferauflage (Edelstahl) [6].

Im silberfarbenen Bestandteil der 1-Euro-Münzen und 2-EuroMünzen findet sich eine Legierung aus 75\% Kupfer und 25\% Nickel. Im goldfarbenen Anteil findet sich eine Legierung mit einer Zusammensetzung aus 5\% Nickel sowie Kupfer und Zink.

Der „Nickelnachweistest“ (Dimethylglyoximtest) an Geldstücken ohne Hautkontakt zeigt einen negativen Nickelnachweis und somit keine Freisetzung von Nickel. Dabei wird dieser Komplexbildner in Anwesenheit von Ammoniak mit dem zu untersuchenden metallischen Gegenstand in Berührung gebracht. Nickelionen lösen eine rosa Farbreaktion aus [5,7].

Schweizer Metallurgen inkubierten Ein- und Zwei-Euro-Münzen eine Woche lang in künstlichem Schweiß. Dabei gaben die Euros viel mehr Nickel ab als der Schweizer Franken. Die gelöste Menge Nickel übertraf den erlaubten Grenzwert für nickelhaltigen Schmuck um das 300fache [8].

In Epikutantestungen bei Nickelallergikern, denen für 48 Stunden 1- und 2-Euro-Münzen auf die Haut aufgebracht wurden, konnte gezeigt werden, dass sich aus Euro-Münzen 240 bis 320-mal mehr Nickel löst, als die EU-Richtlinien (94/27/EG) für Bedarfsgegenstände erlauben.

Der Grenzwert (laut Bedarfsgegenständeverordnung) beträgt maximal $0,5 \mu \mathrm{g} / \mathrm{cm}^{2} /$ Woche [9]. Dies gilt für Modeschmuck, Reißverschlüsse, Uhrengehäuse und andere Produkte, die in direktem und länger dauerndem Kontakt mit der Haut stehen [9].

Dies gilt für Münzen nur deshalb nicht, da sie meist nur kurz mit der Haut in Berührung kommen und nicht als Gebrauchsgegenstände gewertet werden [10].

Ursache der hohen Auslösung von Nickel aus den Euro-Münzen ist die Bimetallstruktur der Münzen, deren äußerer Ring und innerer Kern aus verschiedenen Metalllegierungen bestehen. Während der hellere Teil der Euro-Münzen 25\% Nickel enthält, besteht der dunklere Teil nur zu 5\% aus dem Schwermetall. Dem salzhaltigen Schweiß der Handflächen ausgesetzt, bilden die unterschiedlichen Legierungen ein sog. „Galvanisches Element“.
Im Fall der Euro-Münzen übernimmt das weiße Metall die Rolle des positiven Pols, das gelbe die des negativen Pols. Ähnlich den Vorgängen in einer Batterie wird durch elektrochemische Vorgänge elektrische Energie freigesetzt. Die Legierungen korrodieren und Nickel wird aus den Münzen herausgelöst. Dieser geringe Stromfluss fördert die Freisetzung des allergieauslösenden Metalls, so dass die Geldstücke sogar mehr Nickel abgeben als pures Nickel. Es konnte in künstlichem Schweiß oder gesättigter Kochsalzlösung ein Elektrodenpotenzial bis zu $40 \mathrm{mV}$ nachgewiesen werden [8].

Für die im beschriebenen Fall auftretenden Hautveränderungen spricht das vermehrte Auslösen von Nickel aus den Münzen durch die Hyperhidrosis manuum. Die Menge an Nickel, die von einem Metallobjekt freigesetzt wird, hängt davon ab, ob ein vermehrter Kontakt zu Schweiß vorhanden ist. Aufgrund des Chloridgehaltes und des saureren $\mathrm{pH}$-Wertes des Schweißes kann Nickel in eine lösliche Form gebracht und aus dem Metall herausgelöst werden. Das eigentliche Allergen ist das Nickelion. Nur in der löslichen Form vermag Nickel als Ion die Epidermis zu penetrieren und dort als Metallhapten die Sensibilisierungsreaktion zu starten.

Da bei unserem Patienten von einer Kontaktzeit von über 1 Stunde täglich im Umgang mit Euro-Münzgeld als Bankangestellter mit Kassierertätigkeiten auszugehen war, eine Hyperhidrosis manuum und eine stark verminderte Alkaliresistenz sowie eine atopische Hautdiathese bestand, ist eine berufliche Relevanz der Nickelsensibilisierung anzunehmen. Aufgrund der Tatsache, dass bei unserem Patienten die Nickelallergie erst nach der Einführung des „EURO“ nachzuweisen war, verbunden mit den oben genannten wissenschaftlichen Tatsachen, ergibt sich die Ursächlichkeit der Nickelsensibilisierung durch die Tätigkeit als Kassierer. Das heißt bei einem „nicht relevant nickelfreisetzenden Berufs-/Werkstoff“ wäre diese Sensibilisierung höchstwahrscheinlich vermeidbar gewesen.

Im Verlauf von wenigen Wochen nach Aufnahme des intensiven Hautkontaktes mit den Euro-Münzen kam es erstmalig zu dyshidrosiformen, teils rhagadiformen Hautveränderungen im Bereich der Hände, die im Verlauf zunahmen.

Beim Versuch der Erstellung eines „Tätigkeitsgeprüften Hautschutzplanes“ [11], bei dem der Patient seine Tätigkeit als Kassierer praktisch vor Ort in unserer Klinik unter einem individuellen Hautschutzkonzept, durchführte, zeigte sich eine Verschlechterung des Hautzustandes mit Vermehrung der hyperkeratotisch-rhagadiformen Hautveränderungen bei Exposition gegenüber Euro-Münzgeld. Aufgrund der Tatsache, dass bei unserem Patienten eine Hyperhidrosis manuum vorlag, war offensichtlich selbst unter Hautschutz mittels einer Hautschutzcreme ein allergologisch relevantes Einbringen der Nickelionen in die Haut bei langer Kontaktzeit zu Euro-Münzen möglich.

Im Übrigen zeigten die von uns durchgeführten Epikutantests 2005, also zwei Jahre nach der Erstvorstellung, mit Euro- und DM-Münzen nach 72 Stunden eine positive Reaktion auf beide Münzenarten, da nun schon die geringere Freisetzung von Nickelionen aus den DM-Münzen auszureichen scheint, ein Kontaktekzem zu verursachen. Dies stützt die „Ursächlichkeit“ 
der Nickelsensibilisierung durch den „neuen Berufsstoff“ 1- und 2-Euro-Münzen.

Es bleibt abzuwarten, ob es in den nächsten Jahren wegen des hohen Nickelanteils und der Bimetallstruktur der Euro-Münzen bei gleichzeitiger Hyperhidrosis der Hände gehäuft zu berufsbedingten Handekzemen kommt und sogar eine aktive Nickelsensibilisierung durch diese Münzen möglich ist.

\section{Literatur}

${ }^{1}$ Häberle M. Allergisches Kontaktekzem auf Euro-Münzen. Dermatologie in Beruf und Umwelt 2004; 52: 171 - 175

2 Aberer W. Platitudes in allergy-based on the example of the euro. Contact Dermatitis 2001; 45: 254-255

${ }^{3}$ Barrot R. Ausreichende Nickelexposition als Bedingung für ein Kontaktekzem bei Nickelsensibilisierten. Umwelt und Berufsdermatologisches Bulletin 1999; 83: 1-11

${ }^{4}$ Diepgen TL, Fartasch M, Hornstein OP. Evaluation and relevance of atopic basic and minor features in patients with atopic dermatitis and in the general population. Acta Derm Venereol Suppl (Stockh) 1989; $144: 50-54$

${ }^{5}$ Weiß J, Hoppe U. Der Dimethylglyoximtest. Akt Dermatol 1989; 15: $362-364$

${ }^{6}$ Aberer W, Schöllnast R. Münzen und Nickel - ein Problem? Allergologie 2002; 25: 427-431

${ }^{7}$ Liden C, Carter S. Nickel release from coins. Contact Dermatitis 2001; 44: $160-165$

${ }^{8}$ Nestle FO, Speidel H, Speidel MO. High nickel release from 1 and 2 Euro coins. Nature 2002; 419: 132

${ }^{9}$ European Parliament and Council Directive 94/27/EEC. Official Journal of the European Communities, 22 -07-1994. No L188/1 - 2(nickel).

${ }^{10}$ Aberer W, Holub H. Berufsdermatologische Relevanz der Nickelsensibilisierung. Allergologie 1992; 15: 429-432

${ }^{11}$ Buhles N. Tätigkeitsgeprüfter Hautschutzplan. Derm 2004; 10: $146-$ 148

\section{Buchbesprechung}

\section{Atopische Dermatitis. CD-ROM}

T. Werfel

Stuttgart: Thieme, 2005. 19,95€. ISBN 3-13-133491-6

Prof. Dr. Thomas Werfel von der Medizinischen Hochschule in Hannover ist der Autor einer CD-ROM zur Atopischen Dermatitis mit über 270 Bildern und Tabellen sowie Videos. Mit den Systemvoraussetzungen für den Programmstart wie Windows 2000/XP, mindestens Pentium-III-Prozessor und mehr als 64 MB RAM startet das Programm unkompliziert. Die Gliederung umfasst die Großgruppen Grundlagen, Klinik, Diagnostik, Differenzialdiagnose, Therapie und Präventionsmaßnahmen, jeweils mit weiterer inhaltlicher Untergliederung. Abbildungen und Grafiken beherrschen die Darstellungen, wenige Tabellen und ausreichend klinische Bilder, allesamt in bester Bildqualität. Eingearbeitet sind Animationen zu Calcineurinantagonisten, zu akuter versus chronischer atopischer Dermatitis, zur Hauttestung und Interviews mit Eltern bzw. Patienten. Ein thematischer Komplex startet jeweils mit einer Bildsammlung in der Übersicht, aus der dann das gewünschte „Dia“ angeklickt werden kann. Darstellungen für das Gespräch mit Patienten oder für Laienvorträge sind mit blauem Rand versehen, und nehmen etwa ein Viertel der Bilder ein. Titel des Bildes oder 3-4 Zeilen einer Erläuterung zur Grafik finden sich am unteren Bildrand. Die Befehle „zoomen“ oder „kopieren“ können bei jedem Bild angeklickt werden. Die Videos mit Patientenberichten benötigen den Windows Media Player, der direkt aus der CD-ROM geladen werden kann. Bei Übernahme von Abbildungen aus der Literatur wird die Quelle mit angegeben; bei Videos, die zusammen mit Firmen erstellt wurden, wird der Firmenname genannt. Abbildungen, Grafiken und Schreibstil sind verständlich und berücksichtigen den aktuellen Stand der wissenschaftlichen Erkenntnisse. Die CD-ROM besticht durch die übersichtliche Gestaltung der Programmoberfläche. Das Werk ist vor allem für ärztliche Kollegen für die eigene Fortbildungs- und Vortragstätigkeit wertvoll, aber auch für Nicht-Dermatologen, die mit Neurodermikern arbeiten, z.B. für Kursleiter, Ernährungsfachkräfte, Selbsthilfegruppen und Betroffene.

Christiane Bayerl, Wiesbaden 